\section{PTU-011 IDENTIFYING THE LEARNING NEEDS OF BSW COLONOSCOPISTS USING AN ACTIVE LEARNING DIARY DURING SCREENING LISTS}

${ }^{1} \mathrm{~N}$ Hawkes*, ${ }^{2} \mathrm{~J}$ McDonald. 'Gastroenterology, Cwm Taf University HB, Llantrisant, UK; ${ }^{2}$ Medical Education, Cardiff University, Cardiff, UK

10.1136/gutjnl-2014-307263.85

Introduction In the Bowel Screening Wales (BSW) programme screeni, UKng colonoscopists must meet specified performance criteria and pass both a knowledge-based and practical skills assessment. As such they are highly skilled endoscopists who engage in self-directed learning. We aimed to determine the nature and frequency of ongoing learning opportunities arising on BSW screening lists and practicality of using a structured learning diary. Methods A stratified randomisation identified six BSW colonoscopists (one from each Health Board). A structured diary recording 'active' learning opportunities and self-reflection on learning events (after Knowles) was combined with semi-structured interviews after data collection (minimum 4 BSW lists). A deductive approach to data analysis using a modified grounded theory approach described by Burnard et al. (2008) was used.

Results BSW colonoscopists identified lesion assessment and decision-making (cognitive skills) as the most common learning points. Technical challenges (skills based) and aspects of team performance (attitudinal) were also common. Problems requiring 'situational awareness' or where things were not going to plan (including equipment failure) were less common but prompted more active reflection by colonoscopists. Cumulative entries recorded 35 distinct learning points (some duplicated by more than one colonoscopist) in the following domains; polyp detection and assessment; optimising field of view and access; polyp pre-treatment or lifting; snare selection/technique; diathermy modes and settings; complications; situational awareness and teamwork; judgement and decision-making. All participants felt the data recorded was representative of their normal screening lists (making the learning points transferable to other colonoscopists) and felt that using the diary was feasible and helpful. Participants did not always translate recorded reflections into discernable action plans with specific learning goals mainly due to time constraints - where behaviour changed this was facilitated by local opportunities to discuss with near-peers or during formal endoscopy-based multi-disciplinary meetings. A variety of learning resources were used.

Conclusion The structured learning diary proved to be a practi$\mathrm{cal}$ and useful tool to identify learning opportunities in the context of routine BSW screening lists. Participants identified a number of learning needs - most commonly reported were cognitive skills related to lesion assessment and decision-making. Active reflection promoted by using this kind of tool is most effective when leading to the setting of specific goals and linked to supportive local collaborative working patterns.

\section{REFERENCE}

Burnard P, Gill P, Stewart K, et al. Analysing and presenting qualitative data. Br Dental J 2008;204:429-432.

Disclosure of Interest None Declared.

\section{PTU-012 DEVELOPMENT OF STANDARDS FOR DELIVERY OF TRAINING IN GASTROENTEROLOGY: DEFINING QUALITY AND PROVIDING ACCURATE ASSESSMENT OF UNITS}

${ }^{1} \mathrm{E}$ Britton, 'S Sarkar, ${ }^{2} \mathrm{PK}$ Flanagan*. 'Gastroenterology, Royal Liverpool University Hospital, Liverpool, UK; ${ }^{2}$ Gastroenterology, Arrowe Park Hospital, Wirral, UK
Introduction Training in Gastroenterology is currently defined by the JRCPTB curriculum. Whilst some guidance is provided on composition of clinical sessions its main focus is on overall training structure and expected clinical competencies and knowledge levels. Delivery of training is commonly arranged locally but there are currently no defined standards to describe the expected structure and standard of training within hospital placements. Consequently quality of training can be extremely variable. For the first time we present comprehensive standards detailing the delivery of high quality training in Gastroenterology.

Methods Aims

To develop and validate standards for delivery of training in Gastroenterology.

Methods

Standards were developed by consensus opinion by trainees and consultants including those with relevant subspecialist interests. Refinement of the standards was achieved by further peer review and pilot studies. In all there were 10 domains (inpatients, outpatients, endoscopy, HPB, IBD, luminal, nutrition, GIM, education, teaching) each with 5 possible grades (unacceptable, minimum, average, good, excellent). All points within the lowest grade had to be achieved before higher grade could be awarded. All units were then assessed against the standards with completion of the form by consensus trainee opinion. Overall assessment of individual units was also performed by consensus opinion and using a likert scale.

Results 9 hospitals were assessed and significant variations in training quality were identified with large variation in overall grade attainment (range 22-94\%). Overall grade and $\%$ grade attainment correlated accurately with overall consensus opinion on the relative strengths of units. Poorly performing units were reliably identified and relative strengths of units highlighted. Likert scale assessment was shown to be unreliable with consistently high scores across all units even when overall assessment was poor.

Conclusion Formal standards define how to deliver high quality training, allow objective assessment of units and highlight specific deficiencies in training enabling targeted improvement in delivery. The standards were more reliable than existing methods of assessment. These standards hold the potential to significantly improve training in Gastroenterology in the UK.

Disclosure of Interest E. Britton: None Declared, S. Sarkar: None Declared, P. Flanagan Grant/research support from: Awarded a Shire innovation fund for SpRs.

\section{PTU-013 SIMULATION-BASED HUMAN FACTORS TRAINING IN ENDOSCOPY - PUTTING THE TEAM IN THE SPOTLIGHT}

${ }^{1} \mathrm{~S}$ Webster*, ${ }^{2} \mathrm{~W}$ Howson, ${ }^{2} \mathrm{~A}$ McKay, ${ }^{2} \mathrm{G}$ Smith. ${ }^{1}$ Gastroenterology, West Middlesex University Hospital NHS Trust, UK; ${ }^{2}$ Gastroenterology, Imperial College Healthcare NHS Trust, London, UK

\subsection{6/gutjnl-2014-307263.87}

Introduction Human factors play an important role in adverse incidents and complaints in healthcare. Simulation is increasingly recognised as a safe and effective platform for human factors training. We propose that multiprofessional team-based simulation courses could enhance staff training, staff engagement, patient safety and patient experience in the endoscopy unit.

Methods We designed and delivered four in-situ multiprofessional simulation courses for established endoscopy teams across North West London, using recent clinical incident and complaints 